\title{
Glycemic evaluation of some breads from different countries via in vitro gastrointestinal enzymatic hydrolysis system
}

\author{
Büşra YUSUFOĞLU1 ${ }^{1}$, Mustafa YAMAN², Emine KARAKUŞ ${ }^{1 * ~(D) ~}$
}

\begin{abstract}
Carbohydrates are digested in in vivo gastrointestinal hydrolysis system in the body. To investigate glycemic evaluation of carbohydrate digestion, a lot of in vivo study in the literature, in vitro glycemic study to research postprandial glucose composition with biochemical hydrolysis systems for carbohydrates hasn't been met. In this study, it was investigated glycemic evaluation by using postprandial glucose after digestion of the bread species from different countries such as the United States, Sweden, and Germany with our constructed biochemical hydrolysis system at in vitro conditions. The assays of GI and GL values of these bread species were carried out with the methods based on spectrophotometric. GI and GL values of American white bread (AWB), Sweden white bread (SWB), German white bread (GWB) were calculated by using Turkish white bread (TWB) as reference carbohydrate. AWB, SWB, and GWB have lower GI values than TWB. GL values were also determined for each bread species by using spectrophotometric biochemical in vitro hydrolysis system because the value indicated for consumption of a foodstuff. TWB had higher GL value than the other bread species. GL values of all bread samples were bigger than 20.
\end{abstract}

Keywords: postprandial glucose; in vitro digestion; glycemic index; glycemic load; breads; gastrointestinal system.

Practical Application: Evaluation of Some Breads from Different Countries in terms of Glycemic Index and Glycemic Load.

\section{Introduction}

Carbohydrates in human diet provide the major exogenous source for glucose, which is the primary energy source for cells. The goal of carbohydrate digestion is to break down all complex carbohydrates into monosaccharides for absorption. Digestion begins in the mouth with salivary amylase released during the process of chewing. There is a positive feedback loop resulting in increased oral amylase secretion in people consuming diets high in carbohydrates. The amylase is synthesized in the serous cells of the salivary glands. Amylase breaks starches into maltose and polysaccharides. Amylase is sensitive to $\mathrm{pH}$ and thus is inhibited in the acidic environment of the stomach. Only $5 \%$ of starch is broken down by salivary amylase due to limited exposure. Minimal carbohydrate digestion occurs in the stomach due to the inactivation of amylase in the acidic environment. Pancreatic amylase is released from acinar cells into the small intestine in concert with other enzymes. Amylase targets the $\alpha-1,4$ bonds of complex carbohydrates and is unable to break terminal bonds or $\alpha-1,6$ bonds. Starch is digested in the small intestine to simple components until glucose and other monosaccharides (Hurtado, 2018).

The glycemic index (GI) is a value assigned to foods based on how slowly or how quickly foods cause increases in blood glucose levels. Also known as "blood sugar," blood glucose levels above normal are toxic and can cause blindness, kidney failure, or increase cardiovascular risk. Foods with low glycemic index (GI) scale tend to release glucose slowly and steadily. Foods with a high glycemic index release glucose rapidly. Low GI foods tend to foster weight loss, while foods high on the GI scale help with energy recovery after exercise, or to offset hypo(or insufficient) glycemia. Long-distance runners would tend to favor foods high on the glycemic index, while people with pre- or full-blown diabetes would need to concentrate on low GI foods. Because people with type 1 diabetes and even some with type 2 can't produce sufficient quantities of insulin - which helps process blood sugar-which means they are likely to have an excess of blood glucose. The slow and steady release of glucose in low-glycemic foods is helpful in keeping blood glucose under control (Solanki et al., 2018).

Both the quantity and quality (ie, nature or source) of carbohydrate influence the glycemic response. By definition, the GI compares equal quantities of carbohydrate and provides a measure of carbohydrate quality but not quantity. In 1997 the concept of GL was introduced by researchers at Harvard University to quantify the overall glycemic effect of a portion of food. Thus, the GL of a typical serving of food is the product of the amount of available carbohydrate in that serving and the GI of the food. The higher the GL, the greater the expected elevation in blood glucose and in the insulinogenic effect of the food. The longterm consumption of a diet with a relatively high GL (adjusted for total energy) is associated with an increased risk of type 2 diabetes and coronary heart disease (Foster-Powell et al., 2002).

Postprandial glucose (PPG) concentrations refer to plasma glucose concentrations after eating. Many factors determine the PPG profile. In nondiabetic individuals, fasting plasma glucose concentrations (i.e., following an overnight 8-10 hour fast)

${ }^{1}$ Department of Chemistry, Faculty of Arts and Sciences, Yildiz Technical University, Esenler, Istanbul

${ }^{2}$ Department of Nutrition and Dietetics, Faculty of Health Sciences, Istanbul Sabahattin Zaim University, Halkall, Küçükçekmece, Istanbul

*Corresponding author: eminekaraku@gmail.com 
generally range from 70 to $110 \mathrm{mg} / \mathrm{dl}$. Glucose concentrations begin to rise; $10 \mathrm{~min}$ after the start of a meal as a result of the absorption of dietary carbohydrates. The PPG profile is determined by carbohydrate absorption, insulin and glucagon secretion, and their coordinated effects on glucose metabolism in the liver and peripheral tissues. The magnitude and time of the peak plasma glucose concentration depend on a variety of factors, including the timing, quantity, and composition of the meal. In nondiabetic individuals, plasma glucose concentrations peak; $60 \mathrm{~min}$ after the start of a meal, rarely exceed $140 \mathrm{mg} / \mathrm{dl}$, and return to pre-prandial levels within $2-3$ h. Even though glucose concentrations have returned to preprandial levels by $3 \mathrm{~h}$, absorption of the ingested carbohydrate continues for at least 5-6 h after a meal (American Diabetes Association, 2001).

Digestible carbohydrate is the part of a carbohydrate as "starch and soluble sugars" that can be digested by human enzymes and absorbed directly, providing energy to the body. It does not include dietary fiber which is not digested and can only produce energy through fermentation in the colon. Indigestible carbohydrate is as "mainly hemicellulose and fibre (cellulose)" by the Glycemic Carbohydrate Definition Committee of the American Association for Clinical Chemistry (AACC) (Woolnough et al., 2008).

Bread has been produced by man for thousands of years and is one of few foods common to many societies. The basic concepts of bread and bread making have been modified to suit the prevailing quality of raw materials, the culinary habits and the nature of the society in which it is consumed. Bread and its availability has been fundamental to the political stability of many countries and a sign of prosperity in many cultures. Despite its long history, bread continues to endure and is considered as a staple food in many countries. Bread is a common food over a very wide geographical area. It is eaten almost everywhere that cereals are available and is now being consumed more commonly in regions, such as in South East Asia, that have not been traditional bread consumers. Bread types may be defined based on the constituents of the bread, the shape, or a particular market the bread is aiming to satisfy. The European market provides an example of the range of products that may be found and differing levels of manufacturing sophistication. Most bread is made from wheat, although in northern Europe and Germany bread containing rye is common. In the Latin countries such as Italy, France and Spain, white bread, as baguettes, makes up almost the entire market. Within each country there is also a wide range of breads consumed locally or only at certain times of the year, such as German stollen that is consumed only around Christmas time. Yeast-raised flour-based products also include simple flat forms such as Mexican tortillas, Arabic pitta, pizza bases or Scandinavian flatbread, Chinese steamed bread and a range of sweet goods such as Danish pastries, croissants and doughnuts (Gibson, 2010).

Bread is one of the first baked foods. Archeological digs suggest that bread dates back at least 10,000 years to the Neolithic Period and the Ancient Egyptians. The first bread was made with only flour and water and was unleavened, or without yeast, resulting in the characteristics of a flatbread. Flatbreads are typically dense and difficult to chew, making them hard on teeth and digestion. This is unlike the leavened bread Egyptians were credited with that gained popularity in 5,000 BCE. Leavened bread is much more airy, lighter, and more flavorful because fermentation adds flavors. They are easier to chew and therefore easier to digest (Jenson, 1998).

Simply described, bread is a traditional mixture of flour, water, yeast, and salt combined and baked to create a nourishing food. Societies, cultures, nations, and economies have risen and fallen due to an abundance or lack of it. Bread, a staple of many cultures has been the center of meals and caloric intake for millennia. It was consumed in such large amounts by the Greeks that they were called "artophagoi" or "bread eaters". In the Middle Ages hard slabs of bread were used as edible plates that would soften as the food soaked through it, resulting in the "plate" becoming edible. In present, bread accounts for 25-30\% of daily calories for the average American (Bobrow-Strain). When bread making emigrated from England to America, wheat was one of the first crops to be planted in 1602. The immigrants recognized the importance of this life sustaining crop. They were familiar with it, trusted it and relied on it to recreate the lifestyle they had in their native country. Within a century, acreage dedicated to wheat crops stretched from Maryland to New England (Sardouei-Nasab et al., 2019).

Additionally, bread is the primary source of carbohydrates in most parts of Europe and has also been an important source of whole grain in the Scandinavian countries. From a health perspective, it is important to differentiate between different types of bread because the content can vary widely, from whole-grain rye bread with whole kernels and sourdough to white wheat bread with sifted flour, that is, not whole grain. However, according to a review by Frølich, A man and Tetens most of the wheat and rye consumed in Scandinavia today is in the form of sifted flour. From a public health perspective, in Sweden, as well as in other countries, there is a need to increase the consumption of whole grain especially among young adults. Whole-grain intake is related to several health benefits such as a reduced risk of coronary heart disease, insulin resistance and type 2 diabetes, undesired weight gain, and colorectal cancer. In Sweden, the National Food Agency has suggested five specific dietary recommendations for healthy eating, one of which is to choose primarily whole-grain products when eating cereal food Here whole grain is defined as the whole-grain kernel (endosperm, germ, and bran); the kernel may be ground, crushed or similar but the components should be included in their original proportion for respective grain. Grains refer to wheat, including spelt, rye, oats, barley, maize, rice, millet, sorghum, and other sorghum varieties (Benson, 2013).

As in the world, bread also is one of the most important parts of German cuisine. In Germany the different sorts of bread available can be assigned to four groups according to the different portions of rye and wheat used as cereal sources (Sandvik et al., 2014). The per capita consumption of bread is very diverse across European countries (Table 1).

After AIBI (The International Association of Plant Bakers) information the highest consumption of bread per capital is reported in Turkey (104 kg) and Bulgaria (95 kg), while the lowest one is in Great Britain (approximately $32 \mathrm{~kg}$ ). European 
Table 1. The bread consumption per capita per year in some European countries.

\begin{tabular}{lcc}
\hline \multicolumn{1}{c}{ Country } & Consumption, kg & $\begin{array}{c}\text { Trend with the } \\
\text { previous year }\end{array}$ \\
\hline Turkey & 104.0 & $-10.00 \%$ \\
Bulgaria & 95.0 & Stable \\
Ukraine & 89.0 & $-7.5 \%$ \\
Italy & 68.0 & Stable \\
Russia & 62.0 & $-1.0 \%$ \\
Denmark & 45.0 & Stable \\
Finland & 42.0 & Stable \\
France & 57.0 & Stable \\
Germany & 56.0 & Stable \\
Slovenia & 55.0 & $+2.0 \%$ \\
Belgium & 55.0 & Stable \\
Netherlands & 52.0 & Stable \\
Spain & 42.0 & Stable \\
Great Britain & 32.0 & $-1.0 \%$ \\
Avarage & 59.4 & \\
\hline
\end{tabular}

residents consume on average $59 \mathrm{~kg}$ of bread per year and this level was stable in recent years (Eglite and Kunkulberga, 2017) (Table 1). Although the bread consumption in general is stable, it is important to analyse consumer preferences for bread in order to increase bread consumption by launching successful targeted bread promotions initiatives at national level (Table 1) (Schollenberger et al., 2005).

In this study, it was carried out investigation of postprandial glucose composition to determine glycemic reply of American white bread (AWB), Sweden white bread (SWB), German white bread (GWB) by using Turkish White bread (TWB) as reference carbohydrate with our constructed biochemical enzymatic digestive system in in vitro conditions. The results obtained were compared to each other.

\section{Materials and methods}

\subsection{Reagents and apparatus}

Ekmecik brand Turkish white bread (TWB) and Calandara's Bakery brand American white bread (AWB), golden toast brand German white bread (GWB), Pagen Rosta brand Sweden white bread (SWB) were purchased from the local market in the indicated country.

Pepsin, guar gum, pancreatin, invertase, $\alpha$-amylase, amyloglucosidase (AMG) used for in vitro digestion were obtained from Sigma Chem. Co. (St. Louis, MO). Glucose oxidase/peroxidase D-glucose assay kit with K-GLUC code (GOPOD format) used for determination of glucose in hydrolysate composed after gastrointestinal digestion was purchased Megazyme International Ireland, Bray Business Park, Bray, Co. (Wicklow, IRELAND). Sodium acetate, hydrochloric acid, and ethanol were purchased by Merck (Schuchardt OHG, Hohenbrunn, Germany).

All chemicals used in this study were of analytical grade and were used without further purification. The ethyl alcohol, buffer solutions and all other solutions used for all experiments were prepared with distilled water with a water purification system
(Human Power). All solutions throughout the experiments are mixed with Velp Scientifica Magnetic Stirrers with Hot Plates (India) brand magnetic stirrer and Heidolph Rax model vortex.

The absorbance and $\mathrm{pH}$ measurements were carried out with Shimadzu UVmini-1240 model spectrophotometer and Thermo Scientific 3112000 Star LogR model pH meter (United Kingdom), respectively.

We also used shaking water bath (Nüve ST30, Germany), refrigerator (Samsung Electronics Co., Ltd., Korean), grinder (Sinbo), incubator (Wiseven), precision scales ATX224 Uni Bloc Series (Shimadzu), automatic pipettes (Eppendorf Research) and centrifugate (EBA 20 Hettich Zentrifugen).

\subsection{Methods}

We aimed to compose separately of the mouth, stomach and small intestine in in vivo condition by using our modified Goni`s method (Goñi et al., 1997). We prepared each bread sample before in vitro digestion. First of all, it was determined the percent of digestible carbohydrate amount (DC) in each bread sample. Because the samples have to contain 0.5 gram of digestible carbohydrate in each sample as reference, we calculated how much bread as gram we have to use according to Equation 1 and 2 (Goñi et al., 1997).

$\mathbf{D C}=\mathbf{C}-\mathbf{D F}$

$\mathbf{S}=\frac{0.5}{\mathbf{D C}} \mathbf{X} 100$

DC: The digestible carbohydrate amount as gram

DF: The diet fibre (indigestible carbohydrate) amount of 100gram bread sample written on the package as gram

C: The carbohydrate amount of 100gram bread sample written on the package as gram

S: The bread sample amount used as gram

$0.58 \mathrm{~g}$ of maltose was used as reference carbohydrate for determining the glycemic index of TWB. We calculated the amount of each bread sample containing 0.5 gram of DC by using from Equation 1 and 2. We determined these values as $1.02 \mathrm{~g}$ of TWB, $1.42 \mathrm{~g}$ of AWB, $1.27 \mathrm{~g}$ of SWB, $1.01 \mathrm{~g}$ of GWB. We used TWB as reference carbohydrate in investigation of hydrolysis of the carbohydrate in all other type breads, separately.

\subsection{Postprandial glucose composition with biochemical hydrolysis system}

in vitro biochemical hydrolysis system was constructed to digestion of the carbohydrate in each bread sample. A system was established in the laboratory environment to replace the mouth, stomach and small intestines.

In the mouth digestion (MD) of the carbohydrate in each bread sample; we used a coffee grinder to provide mouth chewing medium. The two shredded pieces of each bread sample and $5 \mathrm{~mL}$ of distilled water were grinded and homogenized separately during 0.5-1 minutes in the coffee grinder at room temperature 
separately. We grinded $1.02 \mathrm{~g}$ of TWB, $1.42 \mathrm{~g}$ of AWB, $1.27 \mathrm{~g}$ of SWB, $1.01 \mathrm{~g}$ of GWB separately. All bread samples were provided to contain 0.5 gram of DC by calculating from Equation 1 and 2 .

In the stomach digestion (SD) of the carbohydrate in each bread sample; each bread sample containing $0.5 \mathrm{~g}$ of digestible carbohydrate was put $50 \mathrm{~mL}$ of falcon tube, added $5 \mathrm{~mL}$ of distilled water and vortexed during 1 minute. $10 \mathrm{~mL}$ of pepsinguar gum solution prepared by adding $100 \mathrm{~mL}$ of $0.05 \mathrm{~N} \mathrm{HCl}$ on $0.5 \mathrm{~g}$ pepsin and $0.5 \mathrm{~g}$ guar gum was added separately to each sample and adjusted $\mathrm{pH}$ to 1.5 and incubated at $37^{\circ} \mathrm{C}$ during $30 \mathrm{~min}$ in a shaking water bath.

To carry out in vitro digestion in the small intestine, we used $136 \mathrm{mg} / \mathrm{mL}$ of pancreatin, $13.4 \mathrm{U} / \mathrm{mL}$ of amyloglucosidase and $25.43 \mathrm{U} / \mathrm{mL}$ of invertase enzymes. To prepare triple enzyme mixture, after the mixture containing $5.44 \mathrm{~g}$ pancreatin and $36.28 \mathrm{~mL}$ of distilled water was centrifuged during 5 minute at $3000 \mathrm{rpm}, 1.78 \mathrm{~mL}$ of amyloglucosidase and $0.00034 \mathrm{~g}$ invertase were added on the supernatant of this mixture. $5 \mathrm{~mL}$ of sodium acetate and $5 \mathrm{~mL}$ of triple enzyme mixture was added on each sample digested in the stomach at 30,60,90, 120 and 180. minutes and incubated at $37^{\circ} \mathrm{C}$ during $30 \mathrm{~min}$ by shaking in a shaking water bath, respectively.

It was taken $0.5 \mathrm{~mL}$ from each sample, added $2 \mathrm{~mL}$ of ethanol and distilled water was added to distilled water until the final volume is $10 \mathrm{~mL}$. We denatured the triple enzymes used in digestion of the small intestine by adding ethanol.

\subsection{Determination of postprandial glucose produced after in vitro enzymatic digestion}

Postprandial D-Glucose was measured in the final reaction medium after in vitro intestinal enzymatic digestion using commercially available glucose oxidase/peroxidase (GOPOD) D-glucose assay kit (GOPOD format) based on enzymatic procedures as colorimetric.

$$
\mathrm{D} \text { - Glucose }+\mathrm{O}_{2}+\mathrm{H}_{2} \mathrm{O} \stackrel{\text { Glucose Oxidase }}{\rightarrow} \mathrm{D} \text { - Gluconate }+\mathrm{H}_{2} \mathrm{O}_{2}
$$

For this aim, $3 \mathrm{~mL}$ of GOPOD reagent containing glucose oxidase and peroxidase was added to $0.1 \mathrm{~mL}$ of each sample taken after incubated during 30,60, 90, 120 and $180 \mathrm{~min}$ and added and incubated at $50{ }^{\circ} \mathrm{C}$ during $20 \mathrm{~min}$. After incubation, the absorbance reading was carried out at $510 \mathrm{~nm}$ via UV spectrophotometer for each sample, separately. The amount of glucose obtained from the final step of in vitro digestion was graphed at five different times between 0 . and 180 . min. This curve is defined as "Hydrolyzed Curve". The area under "Hydrolyzed Curve" (AUHC) was calculated via the excel program.

In this stage, standard glucose assay was also carried out by using $0.1 \mathrm{~mL}$ of D- glucose with GOPOD assay kit. It was also carried out the postprandial D-glucose by applying the same method by using GOPOD D-glucose assay kit after hydrolysing of the carbohydrate in each bread sample, separately.

\subsection{Calculation of Hydrolyzed Index (HI) Value}

The Hydrolyzed Index (HI) value of the postprandial $\mathrm{D}$-glucose obtained from the result of in vitro carbohydrate digestion in each bread sample was calculated from Hydrolyzed Curve between 0 . and 180 . min at 5 different times via excel. HI value of commercial glucose was also calculated by applying the same procedures. Both HI values were calculated according to Equation 4 (Goñi et al., 1997).

$\mathrm{HI}=\frac{\mathrm{AUHC}(\text { Reference Carbohydrate }) *}{\text { AUHC }(\text { Bread Sample })} \times 100$

*While calculating HI values of postprandial D-glucose formation after digestion of carbohydrate in TWB and other bread species, it was used maltose and TWB as reference carbohydrate, respectively.

The calculated HI values of commercial glucose, the postprandial D-glucose obtained from the result of in vitro carbohydrate digestion of TWB, AWB, SWB, and GWB were compared with each other.

\subsection{Investigation of postprandial D-glucose composition in terms of glycemic}

The first step to investigate postprandial D-glucose composition in terms of glycemic, we calculated glycemic index (GI) values for all bread species separately by using TWB as reference carbohydrate. The glycemic index value (GI) of postprandial D-Glucose formed after enzymatic hydrolysis of the carbohydrate in each bread sample by using gastrointestinal enzymes were calculated according to Equation 5 (Goñi et al., 1997).

$\mathrm{GI}=0.7 \mathrm{x}[39.71+(0.549 \mathrm{xHI})$

The second step in order to determine glycemic respect to postprandial D-glucose composition, it was calculated the glycemic load value (GL) of postprandial D-Glucose formed from enzymatic hydrolysis of the carbohydrate in each bread sample by using gastrointestinal enzymes by using the following Equation 6 (Goñi et al., 1997).

$\mathrm{GL}=\frac{\mathrm{DC}}{100} \mathrm{XGI}$

DC: The digestible carbohydrate amount as gram

\section{Results and discussion}

Numerous in vitro carbohydrate digestion methods exist for analysis of the likely glycemic properties of foods. Generally, these methods encompass simulations of oral, gastric and intestinal digestion processes, but the way in which physiological conditions are implemented across methods differs considerably. Some differences are in the mode of comminution, inclusion and duration of a gastric digestion, and choice of amylolytic enzyme. Incubation temperature, $\mathrm{pH}$, duration and stirring mode also differ between methods. Such differences, particularly the method used to mimic chewing, can have a substantial influence on the relative estimate of glycemic potency for a given food. To achieve estimates of high predictive power, and global relevance, a validated, standardised in vitro digestion method must be developed. This article provides a systematic review of 
commonly used and referred to in vitro carbohydrate digestion methods. Methodological discrepancies between protocols are identified thus defining the route a systematic standardisation investigation should take. Since the early 1990s to the present day, an array of in vitro methods for analysing the digestibility of carbohydrate-containing foods have been reported. While generally there is the common theme exhibited in each of these techniques (the imitation of physiological conditions), the ways in which these conditions are simulated across methods differ considerably. A rapid, inexpensive and reliable method of analysis to classify a food within the categories of GI could be beneficial for the South African food industry. The objective of this study was to develop an alternative, less expensive and rapid method to predict glycemic index (GI) in South Africa. The main requirement of the method was that it should accurately and reliably predict the GI of South African foods, at a low cost, within a reduced time frame (Woolnough et al., 2008).

GI concept is using some countries for instance America (Atkinson et al., 2008) Australia, England, South Africa (The 4th edition of the South African Glycemic Index and Load Guide) and some European countries (Gibson, 2010). However, our country has no database related to the GI of foods. Instead of GI database, in Turkey has National Food Composition Database of merely 600 foods (Turkish Food Composition Database, 2020). This database has a glycemic indexes limited number of studies (Löker et al., 2016).

Current global trends relating to type II diabetes incidence make ever more necessary the capacity to predict with accuracy and precision a given food's likely glycemic effect. In June 2006 Glycemic Carbohydrate Definition Committee members expressed concern over the use of in vivo measurements for glycemic prediction due to considerable intra- and inter-individual variability in measured postprandial glycemic responses. The committee members recommended developing a robust and standardised in vitro method that accurately mimics in vivo processes, while measuring a given carbohydrate food's available and glycemic carbohydrate content as indicative of its likely glycemic impact and effect on glycemic response. It is the present lack of a robust, validated, and standardised in vitro carbohydrate digestion method that hinders any large-scale application of the technology to glycemic control via nutrition-labelling in the food industry. To take initial steps toward achieving a standardised method this review compares and contrasts some of the current and commonly referred to in vitro carbohydrate digestion methods. Experimental conditions that differ between methods and which may impact on relative carbohydrate digestibility profiles are exposed, thus defining a path toward the development of a robust, reliable, and standardised in vitro method suitable for large-scale analysis of commercially-digestible carbohydratecontaining foods (Woolnough et al., 2008).

Although a lot of studies have been performed to determine in vivo GI and GL values of foods in Turkey and the world, in vivo determination of them have many disadvantages such as human factors, prolonged determination, inconvenience and high cost (Gibson, 2010). Instead of in vivo analysis, in vitro assays can also be performed to determine the concentration of multiple nutrients at one time. It has not seen any study done in vitro GI and GL determination. in vitro determination of GI and GL values has advantages of being able to determine the GI values of multiple nutrients and to make a large number of bread samples in a much faster and shorter time.

To determine in vivo or in vitro GI value of the food, we should use reference carbohydrates such as maltose, glucose, white bread (Löker et al., 2016).

In this study, after it was determined GI value of Turkish white bread (TWB) taken as reference carbohydrate by using maltose as reference carbohydrate, we determined glycemic parameters values such as GI and GL of (American white bread (AWB), Sweden white bread (SWB), German white bread (GWB) by using Turkish white bread (TWB) as reference carbohydrate.

\subsection{Investigation of postprandial D-glucose composition in terms of glycemic}

Postprandial D-glucose formation and assay procedure after in vitro carbohydrate hydrolysis by using a biochemical hydrolysis system are shown in Figure 1.

To investigate postprandial D-glucose composition after gastrointestinal enzymatic digestion of the carbohydrate in each bread sample in terms of glycemic, first of all, it was plotted the graph as hydrolysed carbohydrate amount as percent against time. Finally, it was determined GI and GL values after calculating the area under the curve (AUHC) of maltose and TWB plottes from Figure 2 (Figure 2, Table 2).

The time-varying curve of carbohydrate amount using maltose as reference carbohydrate during gastrointestinal hydrolysis was called "hydrolysis curve" for TWB (Figure 2). The areas under the hydrolysis curves (AUHC) were calculated separately via excel in both maltose and TWB. Then, the areas under the hydrolysis curve of white bread (AUHC), hydrolysis index (HI), glycemic index (GI) and glycemic load (GL) values of maltose and TWB were calculated, separately (Table 2).

As it was shown in Table 2, the GI value of TWB $(84.70 \pm 3.8)$ was found to be similar to the value stated by the Turkish bread in the Glycemic Index Database of the University of Sydney (Chung et al., 2008). In this way, we have determined the GI value of Turkish white bread which we will use as reference carbohydrates to obtain other bread samples consumed in different countries of the world.

\subsection{Glycemic investigation of different world bread samples hydrolysed in vivo enzymatic system}

To determine glycemic investigation of postprandial glucose composition after with biochemical hydrolysis system our constructed, we calculated GI and GL values of hydrolysed AWB, SWB, and GWB separately. The amount of carbohydrate, fat, protein, and diet fibre of all kinds of bread species used in the study was presented in Table 3 .

The carbohydrate digestion of all bread species from different countries during 180 minute is shown in Figure 3.

When the reaction medium didn't contain glucose because of that bread species have no any digestion of carbohydrate at 0 . 


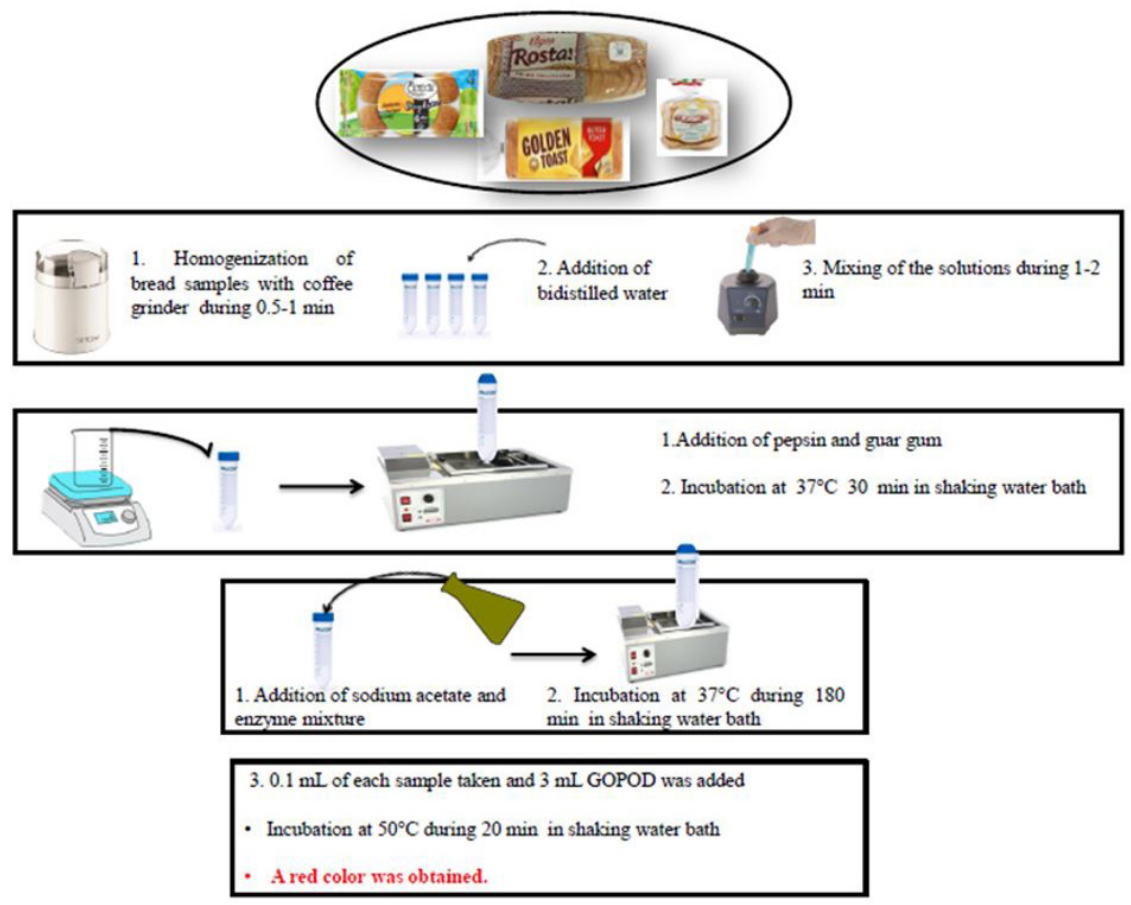

Figure 1. Postprandial D-glucose formation.

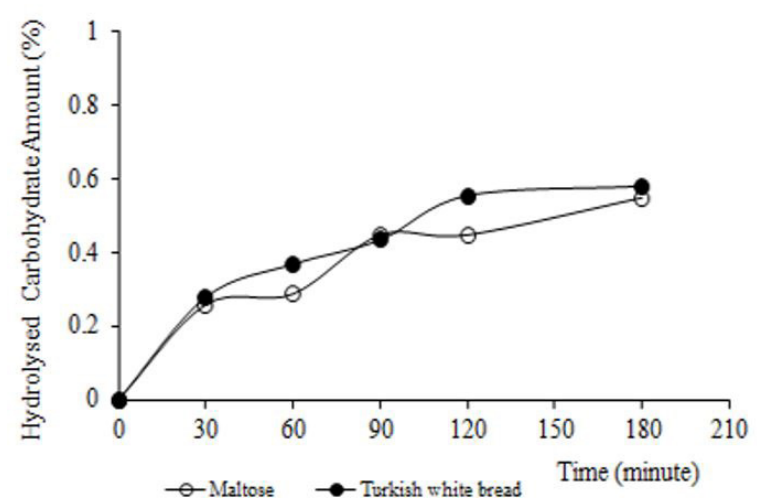

Figure 2. Hydrolysed over-time curve of in vitro carbohydrate hydrolysis using biochemical hydrolysis system our constructed for the determination of the area under the curve (AUHC) for TWB .

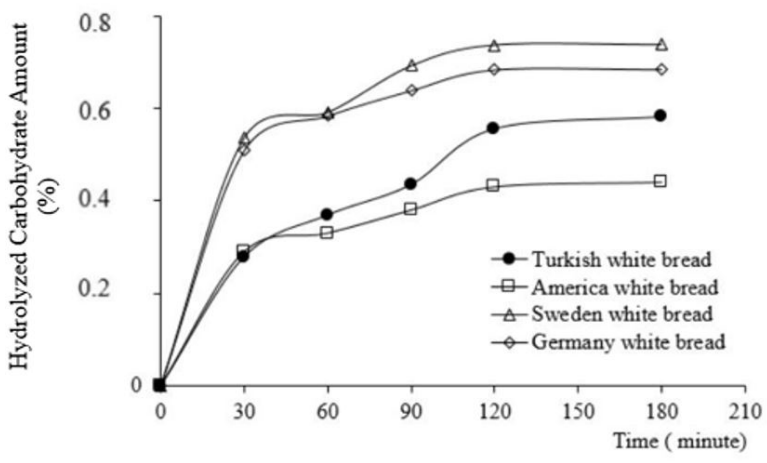

Figure 3. The concentration over-time curves used for the determination of the area under the curve (AUHC) of Turkish white bread (TWB), America white bread (AWB), Sweden white bread (SWB), German white bread (GWB). Turkish white bread was used as reference carbohydrate for calculating GI and GL values of five different bread species.
Table 2. The calculated AUHC, HI, GI and GL values of the hydrolysation of maltose and Turkish white bread (TWB).

\begin{tabular}{lccccc}
\hline $\begin{array}{c}\text { Carbohydrate } \\
\text { Type }\end{array}$ & $\begin{array}{c}\text { Sample } \\
\text { Used (g) }\end{array}$ & AUHC & HI & GI & GL \\
\hline Maltose & 0.58 & $42.2 \pm 1.9$ & $99.7 \pm 4.5$ & $99.7 \pm 4.5$ & $84.7 \pm 3.8$ \\
$\begin{array}{l}\text { Turkish white } \\
\text { bread }\end{array}$ & 1.02 & $59.8 \pm 2.7$ & $140.5 \pm 6.4$ & $84.70 \pm 3.8$ & $41.4 \pm 1.9$ \\
\hline
\end{tabular}

Table 3. The amount of carbohydrate, fat, protein and diet fibre of all kind of bread species.

\begin{tabular}{lcccc}
\hline Bread Sample & Carbohydrate $(\mathrm{g})$ & Fat $(\mathrm{g})$ & Protein $(\mathrm{g})$ & Fiber $(\mathrm{g})$ \\
\hline TWB* & 50.97 & 2.28 & 8.66 & 2.90 \\
AWB & 42.00 & 9.50 & 1.90 & 6.90 \\
SWB & 45.41 & 12.05 & 9.63 & 5.89 \\
GWB & 49.20 & 0.59 & 2.30 & 0.00 \\
\hline *TWB was used as reference carbohydrate for determining the GI and GL values of \\
postprandial D-glucose after enzymatic gastrointestinal hydrolysing for each bread \\
sample.
\end{tabular}

minute. The highest digestion of carbohydrate digestion occurred at 30. minute for all of breads samples. When digestion was continued range from 30 . minute to 180 .minute during 1.5 hours, it was shown that digestion of carbohydrate and hydrolyzed glucose amount were continued to regularly increase in all samples. At the end of 1.5 hours, it is thought that the decrease in digestion may be due to lack of substrate in the reaction medium.

After carbohydrate hydrolysis in each bread sample from different countries used in this study was carried out separately and the amount of postprandial glucose formed as a result of hydrolysis in 30,60,90,120, and 180th minutes was determined as colorimetric. After plotting the hydrolysis curves for each 
bread sample, the area under each hydrolysis curve (AUHC) was calculated separately. HI, GI and GL values of each bread sample were calculated from AUHC values (Table 4).

As seen from in Table 4, the GI and GL levels ranged from $82.0 \pm 3.7$ (TWB) to $54.0 \pm 2.4$ (SWB) and from $39.1 \pm 1.8$ (TWB) to $21.3 \pm 1.0$ (SWB) in different types of bread, respectively. Socio-demographic, geographic, and lifestyle-related factors are associated with what type of bread is consumed (Sandvik et al., 2014). In a study, for 39 foods were expressed on the basis of available carbohydrate content of these foods, highly positive correlations. This study paves the way for the measurements of rapidly digestible starch (RDS), slowly digestible starch (SDS), and resistant starch (RS). Results of quantitative this study, can be used to compare foods eaten on an equal-weight basis (Cummings and Englyst, 1995). Another study shows that a reduction glycemic load linked with reduced body weight (Schuchardt et al., 2016). According to Jiaa et al., have studied physical quality and in vitro starch digestibility of biscuits as affected by addition of soluble dietary fiber from defatted rice bran. Recently, the increasing demand for functional foods has boosted up the food industry to produce novel products such as fiber-enriched, antioxidants, polyphenol, and some mineral and plant based products. In this study was to investigate some features of reformulated biscuits such as rheologic, gelatinization of starch, postprandial blood glucose level, and predicted glycemic index. The objectives of this work properties of the biscuits and exhibit potential to decrease the postprandial blood glucose level (Jia et al., 2020). Recent studies have acknowledged postprandial hypertriglyceridemia as a distinct risk factor for cardiovascular disease. GI and GL have been used as markers of the postprandial glucose and insulin response, and their association with metabolic health and cardiovascular events have been extensively studied with results. Many studies have reported a significant correlation of dietary carbohydrate intake to postprandial lipemia. In a study by Matikainen et al., the consumption of a fructose-sweetened high GL diet for 12 weeks led to a substantial increase in the postprandial triacylglycerol response. Similarly, in another study by Swarbrick et al., a high GL intervention diet, for 10 weeks, led to increased $14 \mathrm{~h}$ postprandial triacylglycerol profiles (Lambadiari et al., 2020). The present invention provides an in vitro analytical method which allows the improved prediction of the glycemic index of a food product from the levels of protein, fat, and sugars/sugar alcohols generated using a simulated digestion of the food product with enzymes. Current glycemic index determination methods generally require human subjects and are both costly and time

Table 4. AUHC, HI, GI and GL values of TWB, AWB, SWB and GWB.

\begin{tabular}{ccccccc}
\hline $\begin{array}{c}\text { Bread } \\
\text { Sample }\end{array}$ & $\begin{array}{c}\text { Sample } \\
\text { Used (g) }\end{array}$ & $\begin{array}{c}\text { RC } \\
\text { Used* }\end{array}$ & AUHC & HI & GI & GL \\
\hline TWB & 1.02 & Maltose & $59.8 \pm 2.7$ & $99.7 \pm 4.5$ & $82.0 \pm 3.7^{\mathrm{a}}$ & $39.1 \pm 1.8^{\mathrm{a}}$ \\
AWB & 1.42 & TWB & $57.1 \pm 2.6$ & $95.2 \pm 4.3$ & $64.3 \pm 2.9^{\mathrm{b}}$ & $22.62 \pm 1.0^{\mathrm{c}}$ \\
SWB & 1.27 & TWB & $41.0 \pm 1.9$ & $67.8 \pm 3.1$ & $54.0 \pm 2.4^{\mathrm{c}}$ & $21.3 \pm 1.0^{\mathrm{c}}$ \\
GWB & 1.01 & TWB & $28.7 \pm 1.3$ & $47.8 \pm 2.2$ & $61.2 \pm 2.8 \mathrm{~b}^{\mathrm{c}}$ & $30.1 \pm 1.4^{\mathrm{b}}$
\end{tabular}

*TWB was used as reference carbohydrate for determining the GI and GL values of postprandial D-glucose after enzymatic gastrointestinal hydrolysing for each bread sample consuming. This method is capable of determining the glycemic index of up to about 15 food samples in one day by one analyst at significantly reduced costs relative to the traditional method using human subjects. Glycemic index values determined using the present inventive method show very high correlation with results obtained using the traditional test using human Subjects. Moreover, this present inventive method can be used with a wide variety of food products, including solid food products as well as semi-solid food products (e.g., yoghurt and the like) and liquid food products (e.g., beverages and the like) (Magaletta \& DiCataldo, 2015).

In general, we have to use carbohydrates such as maltose, glucose and white bread for determining GI and GL values. In this paper, it was used maltose and Turkish White Bread (TWB) to calculate GI value of TWB and the other bread species from different countries, respectively. The aim of this work is to determine the GI value of the food we want by using these references (Goñi et al., 1997). GI and GL values of TWB, AWB, SWB and GWB are shown in Figure 4.

GI value shows maximum the bread samples with high carbohydrate. As can be seen in Figure 4 and Table 4, GI values of world bread types from different countries, the samples with high dietary fiber were observed to be low. It is seen that the dietary fiber amounts of the bread types used are shown as $2.90 \%$ of TWB, $6.90 \%$ of AWB, $5.89 \%$ of SWB, $0.00 \%$ of GWB and GI values of each species decreases with the increase of dietary fiber amount. As a result; except for TWB, other breads AWB, SWB and GWB have lower GI and so they are healthier than TWB. Low glycemic diets have also been reported to result in benefits in weight control because they help control appetite and delay hunger. Low GI diets may also reduce insulin levels and 60 insulin resistance. Recent studies from Harvard School of Public Health report that the risks of diseases such as type 2 diabetes and coronary heart disease are strongly influenced by the glycemic index of the overall diet (Magaletta \& DiCataldo, 2015). The results of this study show the usefulness of the in vitro measurements of glycemic response. When we compared in vivo and in vitro glycemic index; in vitro studies provide many advantages such as experimental time, financial, and ethical burden involved so evaluation of glycemic index instead of human subjects led to in vitro study. Various countries have attached importance to labeling in food products. Brochures, web pages, daily newspaper articles, and measures have taken place in Australia, England, Canada, and South Africa (Gibson, 2010). In 2008, there were 36 products in South Africa with logos

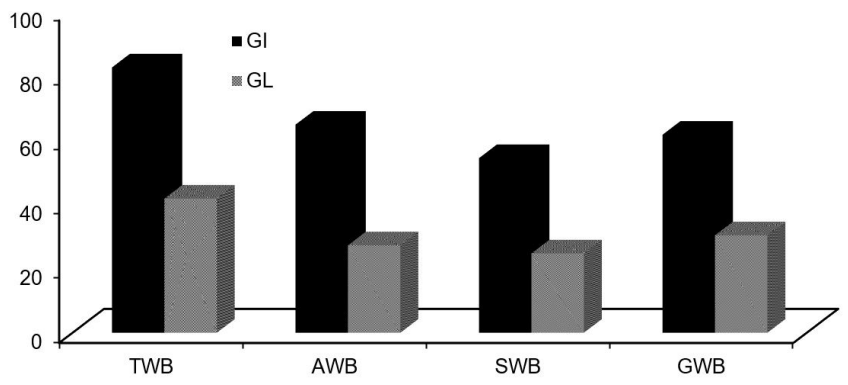

Figure 4. GI and GL values of TWB, AWB, SWB and GWB by using TWB as reference carbohydrate. 
approved by the Glycemic Index Agency of South Africa (GIFSA). Until 2010, 135 products increased in 37 product ranges. Logos approved by the GIFSA for foods: first logo is called "frequent foods" it means low fat and low GI as a coloured light green logo, second logo is called "often foods" it means lower fat and low GI as a coloured dark green logo, third is called "best for exercise" it means lower fat and high GI as a colored red logo, and last is called "special treats" lower fat and intermediate GI coloustared orange logo. According to Venter et al., food labeling knowledge has two purposes; it is the evaluation of the consumer to inform the consumer about the ingredients of the food and to choose a healthy diet. Thus, the consumer must have the correct information about the food that has an impact on metabolism and health (Gibson, 2010).

\section{Conclusion}

Because the amount of food consumed value is also very important as much as the GI of it, GL value indicated for consumption of a foodstuff is important as GI value. GL values of each bread sample is also presented in Figure 4. It is shown that the bread with highest glycemic load is TWB and that of lowest GL is SWB. Especially, the low glycemic index foods are beneficial in management of disease conditions such as diabetes mellitus and cardiovascular disease. COVID-19 pandemic showed to all the world, the matter of glycemic control is primarily precaution even elderly people who have diabetes, cardiovascular disease, and other chronic diseases. The experimental time, financial, and ethical burden involved in the evaluation of glycemic index in human subjects led to in vitro study. In summary, we conclude that identifying in vitro procedure could be useful in the estimation of the GI. Interest in the glycemic index of food products has significantly increased in recent years. The method provides an accurate and inexpensive in vitro method for determining the glycemic index of a wide variety of both food ingredients and finished food products. The present invention provides an in vitro method for determining the glycemic index values for various food products. On the basis of that experiment, our suggest that the indication of a food into high, intermediate or low GI range, as seems possible through the developed in vitro hydrolysis method, would be beneficial during product development, labelling, and consumer reference purposes. It should be remembered that in vitro determined GI values don't always consistently correlate with in vivo determined values, all obtained GI values should be presented to consumers as an indication only. Finally, the GI should not be used as the only criterion to choose healthy foods.

\section{References}

American Diabetes Association. (2001). Postprandial blood glucose. Diabetes Care, 24(4), 775-778. http://dx.doi.org/10.2337/diacare.24.4.775. PMid:11315848.

Atkinson, F. S., Foster-Powell, K., \& Brand-Miller, J. C. (2008). International tables of glycemic index and glycemic load values. Diabetes Care, 31(12), 2281-2283. http://dx.doi.org/10.2337/dc081239. PMid:18835944.

Benson, A. (2013). The Rise and Fall of Bread in America Honors Theses (Paper 11). Johnson \& Wales University. Retrieved from https:// citeseerx.ist.psu.edu/viewdoc/download?doi=10.1.1.675.2079\&re $\mathrm{p}=$ rep $1 \&$ type $=$ pdf

Chung, H. J., Shin, D. H., \& Lim, S. T. (2008). in vitro starch digestibility and estimated glycemic index of chemically modified corn starches. Food Research International, 41(6), 579-585. http://dx.doi.org/10.1016/j. foodres.2008.04.006.

Cummings, J. H., \& Englyst, H. N. (1995). Gastrointestinal effects of food carbohydrate. The American Journal of Clinical Nutrition, 61(4, Supplupl. Suppl), 938S-945S. http://dx.doi.org/10.1093/ ajcn/61.4.938S. PMid:7900692.

Eglite, A., \& Kunkulberga, D. (2017). Bread choice and consumption trends. In Baltic Conference on Food Science and Technology FOODBALT "Food for consumer well-being" (Vol. 11, pp. 178-182). Jelgava, Latvia: Latvia University of Life Sciences and Technologies.

Foster-Powell, K., Holt, S. H. A., \& Brand-Miller, J. C. (2002). International table of glycemic index and glycemic load values: 2002. The American Journal of Clinical Nutrition, 76(1), 5-56. https://doi.org/10.1093/ ajcn/76.1.5.

Gibson, N. (2010). Development of a rapid assessment method for the glycaemic index (Master's thesis). University of Pretoria, Pretoria.

Goñi, I., Garcia-Alonso, A., \& Saura-Calixto, F. (1997). A Starch Hydrolysis Procedure to Estimate Glycemic Index. Nutrition Research (New York, N.Y.), 17(3), 427-437. http://dx.doi.org/10.1016/S02715317(97)00010-9.

Hurtado, C. W. (2018). Carbohydrate digestion and absorption (Naspghan Physiology Series). Retrieved from https://www.naspghan.org/files/ documents/pdfs/training/curriculum-resources/physiology-series/ Carbohydrate_digestion_NASPGHAN.pdf.

Jenson, I. (1998). Bread and baker's yeast. In B. J. B. Wood (Eds.), Microbiology of fermented foods (Chap. 7, pp. 172-198). Boston, MA: Springer. Retrieved from https://link.springer.com/ chapter/10.1007/978-1-4613-0309-1_7

Jia, M., Yu, Q., Chen, J., He, Z., Chen, Y., Xie, J., Nie, S., \& Xie, M. (2020). Physical quality and in vitro starch digestibility of biscuits as affected by addition of soluble dietary fiber from defatted rice bran. Food Hydrocolloids, 99, 105349. http://dx.doi.org/10.1016/j. foodhyd.2019.105349.

Lambadiari, V., Korakas, E., \& Tsimihodimos, V. (2020). The impact of dietary glycemic index and glycemic load on postprandial lipid kinetics, dyslipidemia and cardiovascular risk. Nutrients, 12(8), 2204. http://dx.doi.org/10.3390/nu12082204. PMid:32722053.

Löker, G. B., Yaman, M., Çevikkalp, S. A., Basaran, N., Özalp, Ç., Ünlü, S. G., Gültekin, D. D., Çalık, S., Öztürk, M., Altay, H., Türker, I., Ceyhan, H., Güngör, A. A., \& Amoutzopoulos, B. (2016). Food composition of fruits in Turkey: research outputs from the new Turkish Food Composition Database, TürKomp. Fruits, 71(6), 419-431. http://dx.doi.org/10.1051/fruits/2016031.

Magaletta, R. L., \& DiCataldo, S. N. (2015). U.S. Patent No. 9,109,247. Washington, DC: U.S. Patent and Trademark Office.

Sandvik, P., Kihlberg, I., Lindroos, A. K., Marklinder, I., \& Nydahl, M. (2014). Bread consumption patterns in a Swedish national dietary surveyfocusing particularly on whole-grain and rye bread. Food \& Nutrition Research, 58(1), 24024. http://dx.doi.org/10.3402/fnr. v58.24024. PMid:25278822.

Sardouei-Nasab, S., Mohammadi-Nejad, G. \& Nakhoda, B. (2019). Yield stability in bread wheat germplasma cross drought stress and non-stress conditions. American Society of Agronomy, 111(1), 175181. https://doi.org/10.2134/agronj2018.06.0381.

Schollenberger, M., Drochner, W., Rufle, M., Suchy, S., Terry-Jara, H., \& Muller, H. M. (2005). Trichothecene toxins in different groups 
of conventional and organic bread of the German market. Journal of Food Composition and Analysis, 18(1), 69-78. http://dx.doi. org/10.1016/j.jfca.2003.10.009.

Schuchardt, J. P., Wonik, J., Bindrich, U., Heinemann, M., Kohrs, H., Schneider, I., Möller, K., \& Hahn, A. (2016). Glycemic index and microstructure analysis of a newly developed fiber enriched cookie. Food \& Function, 7(1), 464-474. http://dx.doi.org/10.1039/ C5FO01137J. PMid:26514289.

Solanki, S., Suchak, C., Thakkar, P., Thakkar, S., Patel, R., Chaudhary, J., Ahmad, F., Thakkar, V., Parihar, A., Trivedi, R., \& Sen, D. J. (2018).
Reflection of Glycated Haemoglobin (Hba1c) on Glycemic Index (GI) during diabetes. European Journal Pharmaceutical and Medical Research, 5(6), 295-301.

Turkish Food Composition Database (2020). Retrieved from http:// www.turkomp.gov.tr/main.

Woolnough, J. W., Monro, J. A., Brennan, C. S., \& Bird, A. R. (2008). Simulating human carbohydrate digestion in vitro: a review of methods and the need for standardisation. International Journal of Food Science \& Technology, 2(43), 2245-2256. http://dx.doi. org/10.1111/j.1365-2621.2008.01862.x. 